\title{
Classifying weak and strong components using ROC analysis with application to burn-in problem
}

\author{
Shaomin $\mathrm{Wu}$, and Min Xie, Fellow, IEEE* ${ }^{* \dagger}$
}

\begin{abstract}
Any population of components produced by manufacturer might be composed of two sub-populations: weak components in a small proportion are less reliable, and deteriorate faster, whereas strong components in a large proportion are more reliable, and deteriorate slower. When selecting an approach to classifying the two sub-populations, one could build a criterion aiming at minimizing the expected mis-classification cost due to mis-classifying weak (strong) components as strong (weak). However, in practice, the unit mis-classification cost, such as the cost of mis-classifying a strong component as weak, can not be estimated precisely. Minimizing the expected mis-classification cost becomes more difficult. This problem is considered in this paper by using ROC (Receiver Operating Characteristic) analysis, which is widely used in medical decision making community to evaluate the performance of diagnostic tests, and in machine learning to

\footnotetext{
${ }^{*}$ Corresponding author: Shaomin Wu is with The University of Reading, Whiteknights, Reading, RG6 6AW, UK. E-mail: shaomin.wu@reading.ac.uk. Telephone: +44 118378 7187; Fax: +44 1189313856

${ }^{\dagger}$ Min Xie is with Department of Industrial and Systems Engineering, National University of Singapore, Singapore 119077, E-mail: mxie@nus.edu.sg
} 
select among categorical models. The paper also uses ROC analysis to determine the optimal time for burn-in in order to remove the weak population. The presented approaches can be used for the scenarios when the following information cannot be estimated precisely:

(1) life distributions of the sub-populations,

(2) mis-classification cost, and

(3) proportions of sub-populations in the entire population.

Index Terms - Mixed distribution, classification, Receiver Operating Characteristic (ROC) analysis, burn-in.

\section{NOMENCLATURE}

$\alpha(t)$ probability of mis-classifying a strong component to be weak at time $t$

$\beta(t)$ probability of mis-classifying a weak component to be strong at time $t$

$C_{\alpha}$ cost of mis-classifying a positive (strong) observation to be negative (weak)

$C_{\beta}$ cost of mis-classifying a negative (weak) observation to be positive (strong)

$p$ proportion of the negative (or weak) observations in the entire population

$t_{k}$ check time in a burn-in test, where $k=1, \ldots, n$

\section{Introduction}

This paper utilizes receiver operating characteristic (ROC) analysis to select optimal approaches to distinguish weak \& strong components in a population 
when precise information about the life distribution of sub-populations, and mis-classification costs in the entire population might not be available. It is well-known that components produced by a manufacturer can be composed of two sub-populations: weak \& strong. The weak components in a small proportion are less reliable, and deteriorate faster, whereas the strong components in a large proportion are more reliable, and deteriorate slower. In order to improve the reliability of such populations, the weak components need weeding out from the entire population. This can be achieved by many approaches, from which people can select optimal ones. Burn-in is such a method commonly used in electronics industry. In selecting such an approach, one could minimize the expected mis-classification cost incurred due to mis-classifying weak (strong) components as strong (weak). However, in practice, the unit mis-classification cost, such as the cost on mis-classifying a strong component as weak, can not be estimated precisely. Minimizing the expected mis-classification cost becomes difficult. In the decision making community, there often exist classification problems where the outcomes are binary. For example, doctors usually have to establish whether a patient carries a kind of virus (negative) or not (positive). Such a decision may lead to two types of mistake: mis-classifying a positive (negative) to be negative (positive), which are known as type-I \& type-II errors in statistics. These mistakes incur mis-classification cost. In order to select optimal models with the minimum mis-classification cost, one needs information such as the proportion of the negative (or positive ) patients - aka, class distribution, and unit mis-classification cost. When such information is not available, ROC analysis can be used to select optimal models, and discard suboptimal ones 
independently from (and prior to specifying) the cost context or the class distribution (see [1] for a comprehensive overview).

ROC analysis is related in a direct \& natural way to cost analysis of diagnostic decision making. It is derived from signal detection theory [2] where it is used to determine if an electronic receiver is able to satisfactorily distinguish between signal \& noise. It has been used in medical imaging \& radiology [3], psychiatry [4], non-destructive testing [5], and machine learning [6].

Burn-in is a widely used approach for weeding out weak components before they are delivered to customers. The components that fail during the burn-in procedure will be scrapped or repaired, and only those that survived the burn-in time will be considered to be of good quality.

A burn-in procedure is commonly associated with three steps: estimating a lifetime distribution for a given population, assessing the suitability of a burn-in procedure based on the property of the lifetime distribution, and selecting a burn-in optimization criterion in order to determine the duration of the burn-in. Burn-in optimization criteria usually include performancebased methods [7, 8], and cost-based methods [9, 10]. For a given population, a performance-based optimization criterion might be either to maximize the mean residual life [7], or to achieve pre-defined reliability target [8]. A costbased optimization criterion is to minimize a cost function considering all kinds of costs associated with the burn-in procedure [9, 10]. For some other recent research on burn-in, see, e.g., [11, 12, 13.

The cost-based optimization criteria are usually chosen to determine the optimal burn-in time for a mixture of two sub-populations. Two-mixed dis- 
tributions in the reliability literature are usually used to model the lifetime of a mixture of sub-populations. For example, a two-mixed Weibull distribution was applied to fit failure data of electronic tubes by Kao [14], Boardman \& Colvert [15], and Kim [16]; a two-mixed exponential distribution was studied by Perlstein et. al. [17, [18].

A burn-in procedure for such populations may cause the following burn-in errors: a strong (weak) component is classified as a weak (strong ) component. Some researchers determine optimal burn-in times by targeting at minimising the cost due to the burn-in errors. Kim [16] defines a criterion considering the costs of burn-in errors for a two-mixed Weibull distribution. Tseng et al. [19], Tseng \& Tang [20], and Tseng \& Peng [21] use Wiener processes to describe the continuous degradation path of the quality characteristic of highly reliable products, with a consideration of the costs of burn-in errors. In order to obtain the optimal burn-in times, they assume that the following information is given:

(A) mis-classification cost for an individual component, or called unit misclassification cost, and

(B) proportion of the sub-population (weak \& strong), or called class distribution in the following.

The unit mis-classification cost in (A) is commonly associated with the various costs including replacement (or repair) cost, and maybe warranty cost. Therefore, precisely estimating the unit mis-classification cost can be very difficult. In the case the unit mis-classification cost is randomly distributed, using the approaches introduced by [16, 19, 20, 21] to determine 
the optimal burn-in time will become impossible. Furthermore, precisely estimating the class distribution in (B) can be questionable in practice.

This paper presents approaches to classifying weak components from strong components. It utilizes ROC analysis to determine the optimal burnin times for the scenarios where information on mis-classification costs \& class distribution are not available.

The paper is structured as follow. Section 2 introduces concepts of the ROC space, ROC curves, and the area under the ROC curve. Section 3 presents a new cost model considering mis-classification costs, and discusses the cost models derived by [19, 20, 21]. Section 4 introduces novel approaches to obtaining optimal burn-in times when the cost context and/or class distribution are not available. Section 5 studies a case originally from [20, 21]. Section 6 concludes our findings of the paper.

\section{ROC analysis}

In classifying whether a patient carries a kind of virus, a model (or doctor) can choose one decision from four possible ones which consist of two types of errors when comparing the model result with the real situation of a patient as shown in Table 1. If both the real situation and model are positive, it is called a true positive (TP). If the real situation is positive whereas the model is negative, it is called a false negative (FN). False positive (FP) \& true negative $(\mathrm{TN})$ are defined similarly.

Define

$T_{r}=\frac{\text { the number of true positives }}{\text { the number of true positives }+ \text { the number of false negatives }}=\frac{\mathrm{TP}}{\mathrm{TP}+\mathrm{FN}}$, 
Table 1: Contingency table

\begin{tabular}{|c|c|c|c|}
\hline & \multicolumn{2}{|c|}{ Actual situation } \\
\hline & & Positive & Negative \\
\hline & Positive & TP (True Positives) & FP (False Positives) \\
\hline result & Negative & FN (False Negatives) & TN (True Negatives) \\
\hline
\end{tabular}

and

$F_{r}=\frac{\text { the number of false positives }}{\text { the number of true negatives }+ \text { the number of false positives }}=\frac{\mathrm{FP}}{\mathrm{TN}+\mathrm{FP}}$.

For any decision, we hope that their $F_{r}$ 's are small, and $T_{r}$ 's are large, which is a two criterion optimization problem. Instead of optimizing the two-criterion problem, one can minimize the following cost function:

$$
C_{\alpha}(1-p)\left(1-T_{r}\right)+C_{\beta} p F_{r}
$$

For example, given a dataset containing 100 patients, 80 of whom do not carry the virus (positive), and the rest do (negative). Two models, say, A \& B, are developed to classify the patients. We have the following results for the two models.

- Model A classifies 70 positives \& 12 negatives correctly, which means $\mathrm{TP}=70, \mathrm{TN}=12, \mathrm{FP}=8$, and $\mathrm{FN}=10$. Then we have

$$
\begin{aligned}
& -F_{r}=\mathrm{FP} /(\mathrm{FP}+\mathrm{TN})=8 / 20, \text { and } \\
& -T_{r}=\mathrm{TP} /(\mathrm{TP}+\mathrm{FN})=70 / 80 .
\end{aligned}
$$

- Model B classifies 65 positives \& 16 negatives correctly, which means $\mathrm{TP}=65, \mathrm{TN}=16, \mathrm{FP}=4$, and $\mathrm{FN}=15$. Then, we have

$$
-F_{r}=\mathrm{FP} /(\mathrm{FP}+\mathrm{TN})=4 / 20, \text { and }
$$




$$
-T_{r}=\mathrm{TP} /(\mathrm{TP}+\mathrm{FN})=65 / 80 .
$$

According to (1), when values $p, C_{\alpha}$, and $C_{\beta}$ are available, for example, one can choose Model $\mathrm{B}$ if $\frac{10}{80} C_{\alpha}(1-p)+\frac{8}{20} C_{\beta} p>\frac{15}{80} C_{\alpha}(1-p)+\frac{4}{20} C_{\beta} p$. Otherwise, model A will be selected.

However, the values $p, C_{\alpha}$, and $C_{\beta}$ might be unknown, hence, one might hope to choose optimal models simply based on the values of $T_{r} \& F_{r}$. This goal can be achieved by using ROC analysis,.

ROC analysis studies positions of points or curves in a two-dimensional space, called ROC space, in which $T_{r}$ 's are plotted on the $\mathrm{Y}$ axis \& $F_{r}$ 's on the $\mathrm{X}$ axis. Each point (or curve if one links the points, which is shown as follow) in the space represents a model. The ROC space depicts relative trade-offs between benefits (true positives) and costs (false positives). ROC analysis is usually associated with model selection when both the class distribution and mis-classification cost distribution are unknown at the model development time.

On the basis of their outputs, classification models can be categorized into:

Discrete model: If a model classifies a positive (negative) observation to either positive or negative, it is called a discrete model.

Continuous model: For a given sample with $n$ observations, if a model classifies all of the $n$ observations to be positive with predicted scores $\nu_{i}\left(0 \leq \nu_{i} \leq 1 \& i=1, \ldots, n\right)$, it is called a continuous model.

A discrete model only has one $F_{r} \&$ one $T_{r}$; it can therefore be represented 
by a point in the ROC space (see Figure 11 For a continuous model, a cutoff point can divide the predicted scores of the model into two parts: positive or negative. For example, set a cutoff point to be 0.5 , the observations with predicted scores larger than 0.5 are positive, otherwise negative, and the model with this cutoff point can be represented by a point in the ROC space. Apparently, different cutoff points create different points in the ROC space, hence, a continuous model can produce many points on the ROC space with different $F_{r}$ 's \& $T_{r}$ 's. Sequentially linking all of the points, one can draw a curve (see Figure 2). As a consequence, a continuous model is represented by a curve in the ROC space.

In the following, approaches to selecting optimal models for both discrete and continuous models are presented.

\subsection{Discrete Models}

Figure 1 shows the ROC space with five discrete models: $\mathrm{A}(0.3,0.6), \mathrm{B}(0.1$, $0.7), \mathrm{C}(0.4,0.4), \mathrm{D}(0.34,0.9)$, and $\mathrm{E}(0.6,0.9)$.

We call the convex hull constructed by the points in the ROC space the ROC convex hull (ROCCH) of the corresponding set of models. Figure 3 shows the ROC curve of models A, B, C, D, and E. Model C is clearly not optimal. Perhaps surprisingly, model E can never be optimal because it does not lie on the convex hull, according to the following Lemma [6].

Lemma 1. For any set of cost $\&$ class distributions, $F_{r}, T_{r}, C_{\alpha}, C_{\beta}$, there is a point on the ROCCH with minimum expected cost.

\footnotetext{
${ }^{1}$ Aside from Figure 6 in all of figures in this paper, $\mathrm{X}$-axes represent $F_{r}$ 's, and Y-axes represent $T_{r}$ 's.
} 


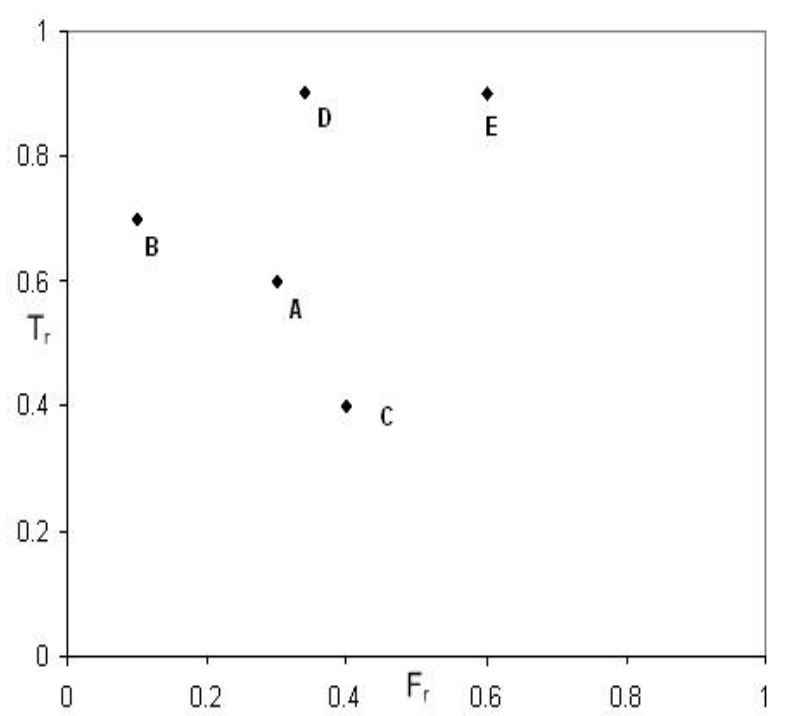

Figure 1: An ROC space with five models in.

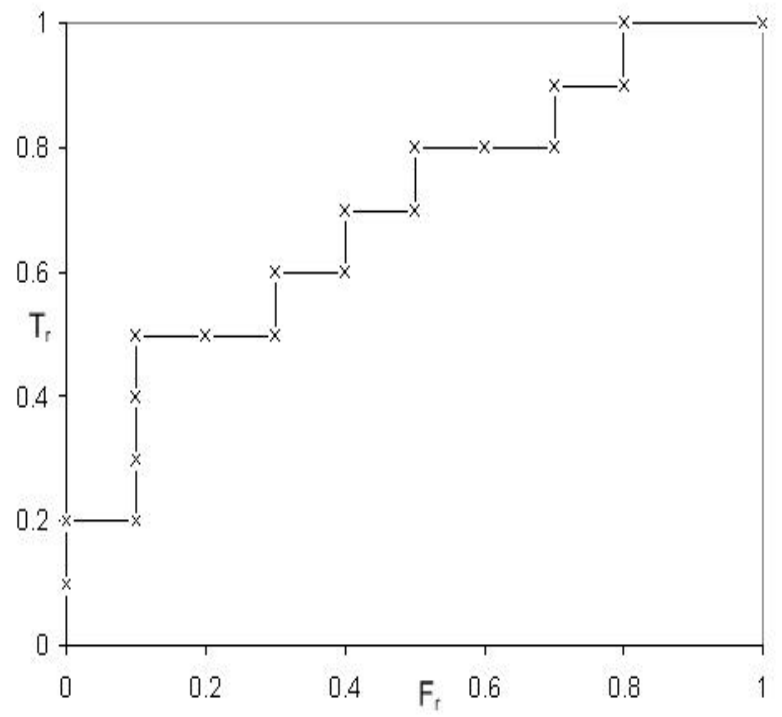

Figure 2: An ROC curve for a continuous model.

From the above Lemma, only the points (or models) on the ROCCH are optimal. For example, in Figure 3, models B \& D are potentially optimal. Now the problem is how to select the optimal model from Model B \& Model 


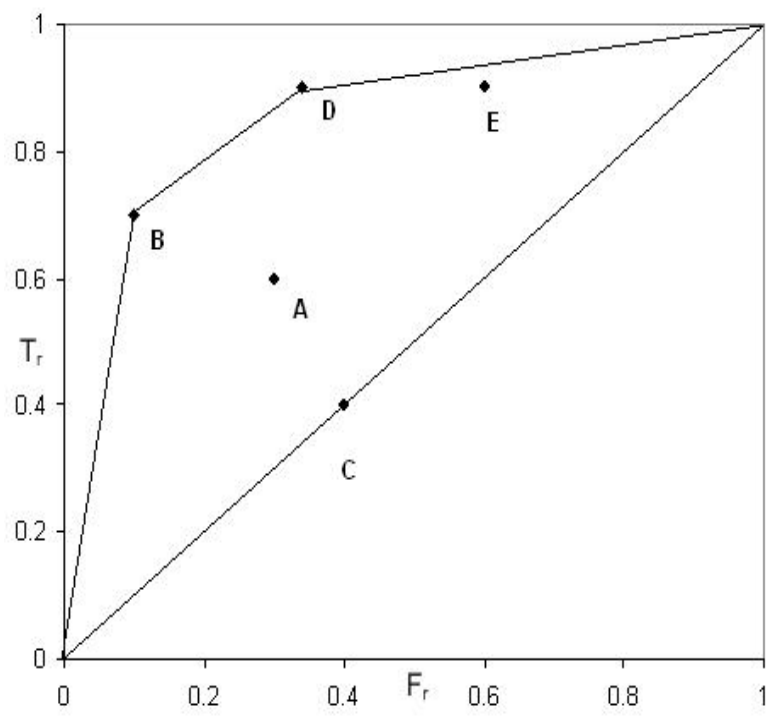

Figure 3: ROC convex hull of models A, B, C, D, and E.

D. A commonly used method in the ROC analysis is to choose the one with the largest area of rectangle constructed by the point representing the model, points $(0,0),(1,0)$, and $(1,1)$. Figure 4 shows the area constituted by model B. If we assume $C_{\alpha}(1-p)=C_{\beta} p$, then comparing the rectangles areas (which equals to $\left.0.5\left(1+T_{r}-F_{r}\right)\right)$ built by different models, is equivalent to comparing the values $C_{\alpha}(1-p)\left(1-T_{r}\right)+C_{\beta} p F_{r}$.

\subsection{Continuous models}

For a continuous model, let $x$ denote the values of $F_{r}$ 's \& $1-y$ denote the values of $T_{r}$ 's. Suppose there is a relationship: $y=f(x)$. Then the expected cost in $x$ of (1) can be given by

$$
\begin{aligned}
& \int_{0}^{1}\left(C_{\alpha}(1-p)(1-y)+C_{\beta} p x\right) d x \\
= & C_{\alpha}(1-p)+\frac{1}{2} C_{\beta} p-C_{\alpha}(1-p) \int_{0}^{1} f(x) d x .
\end{aligned}
$$




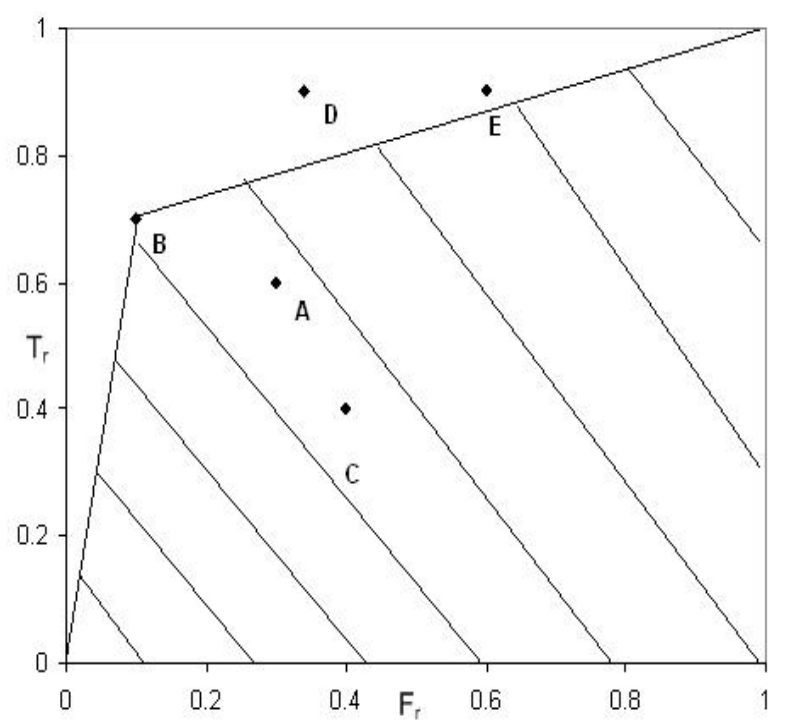

Figure 4: AUC of model B.

Therefore, in case $p, C_{\alpha}, C_{\beta}$ are not available, one can compare values $\int_{0}^{1} f(x) d x$, which defines the Area Under the ROC Curve (AUC). Apparently, a good model should have a large AUC. AUC $=1$ means there is a cutoff point such that the corresponding classification model has a 100\% accuracy. The shadowy area in Figure 5 is approximately equivalent to the integral $\int_{0}^{1} f(x) d x$ by the Trapezoidal integration rule.

In sum, the main approaches to comparing models using ROC analysis are as follows.

- Among discrete models, only models on the convex hull are optimal models. When no information about unit mis-classification cost \& class distribution are given, a model - on the ROC convex hull-with the largest area of the rectangle constructed by the model itself, points $(0,0),(1,0)$, and $(1,1)$ is the optimal model. 


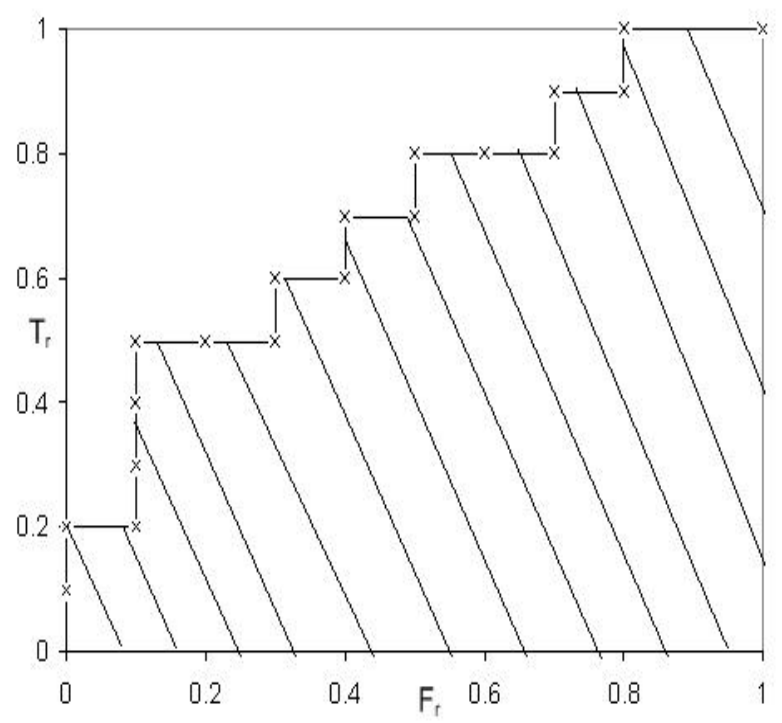

Figure 5: AUC of the ROC curve of a continuous model.

- Among continuous models, a model with the largest AUC is the optimal model.

The above approaches can be utilized in the case when weak \& strong components need be classified.

There is considerable research on ROC analysis in both statistics in medicine, and machine learning. For a more comprehensive understanding of ROC analysis, the reader is referred to Provost \& Fawcett [6], Flach \& $\mathrm{Wu}$ [22], Wu \& Flach [23] in machine learning and data mining, and Zweig \& Campbell [24] in medical decision making community.

\section{A cost model for burn-in optimization}

Suppose a population is composed of two sub-populations: a weak population with a proportion of $p$, and a strong population with a proportion of $1-p$. Suppose that $t_{1}, \ldots, t_{n}\left(t_{1}<t_{2}<\ldots<t_{n}\right)$ are the check times in a burn-in test: 
each product in the test is checked at time point $t_{k}(k=1, \ldots, n)$. If a product is checked failed, it is removed from the test; otherwise, it will continue to stay in the test. The expected mis-classification cost, which includes the costs of mis-classifying a strong product as weak, and mis-classifying a weak product as strong, is given by

$$
C(t)=C_{\alpha}(1-p) \alpha(t)+C_{\beta} p \beta(t)
$$

where $\alpha(t)=1-T_{r}, \beta(t)=F_{r}$.

The contingency table of the decision at burn-in time $t$, is shown in Table 2.

Table 2: Contingency table.

\begin{tabular}{ccc}
\hline Burn-in results & Strong population & Weak population \\
\hline Survive & $1-\alpha(t)$ & $\beta(t)$ \\
Fail & $\alpha(t)$ & $1-\beta(t)$ \\
\hline
\end{tabular}

The model in (2) has been utilized by [16, 19, 20, 21].

Denote

$$
L(t)= \begin{cases}g_{1}(t)+\sigma B(\tau(t)) & \text { for weak products } \\ g_{2}(t)+\sigma B(\tau(t)) & \text { for strong products }\end{cases}
$$

where $g_{2}(t)>g_{1}(t)>0, g_{i}(t)=\exp \left\{-\eta_{i} t^{\delta}\right\}(i=1,2), \tau(t)=t^{\gamma}$, and $B($. denotes the standardized Brownian motion.

Tseng \& Tang [20], and Tseng \& Peng [21] classify the strong products from the weak ones, considering the following conditions: the NCD (Noncumulative Degradation) \& CD (Cumulative Degradation).

The NCD method [20] classifies a product to be strong at time $t$ if

$$
G_{0}(t) \geq \xi_{0}(t)
$$


where $G_{0}(t)=L(t)$.

The CD method [21] classifies a product to be strong at time $t$ if

$$
G_{1}(t) \geq \xi_{1}(t)
$$

and $G_{1}(t)=\int_{0}^{t} L(s) d s$.

Both $\xi_{0}(t)$ in (4) and $\xi_{1}(t)$ in (5) are obtained by minimizing the misclassification cost in (2).

However, there exist some weaknesses in the work of Kim [16], and Tseng et al. [20, 21].

- They all assume that precise mis-classification costs, $C_{\alpha} \& C_{\beta}$ in (2), are given. However, in practice, it is rare for the costs of mis-classifying components to be known precisely. For example, mis-classifying a weak component as strong might incur warranty cost, and business losses due to the failure within operating time. As these costs evolve over time, it is hard to estimate them precisely.

- Kim [16] assumes that the life distributions of the weak \& strong population can be estimated. However, due to technological innovation, and products becoming more reliable, there might not be enough failures during the burn-in test for estimating the life distributions of weak \& strong populations.

Therefore, there is a need to determine the optimal burn-in time for the scenarios where the mis-classification costs, and life distributions might not be specified. 
In what follows, assume the burn-in procedure: at time $0, M$ components are put into a burn-in environment, where $M=M_{n}+M_{w}, \& M_{n}\left(M_{w}\right)$ is the size of the strong (weak) population. At a check time point $t_{k}(k=1,2, \ldots, n)$, $m_{k}$ (or $w_{k}$ ) components from the strong (or weak) population are found failed until time $t_{k}$, and $\left(m_{k}+w_{k}\right) \leq M$, which means, $\beta\left(t_{k}\right)=1-\frac{w_{k}}{M_{w}}$, and $\alpha\left(t_{k}\right)=\frac{m_{k}}{M_{n}}$. With the burn-in time progresses, data collected at $t_{k}$ $(k=1, \ldots, n)$ accumulate; hence, the life distributions of both strong \& weak components estimated at $t_{k}, k=1, \ldots, n$, can be different, see [21] as an example. Assume that the life distribution of strong (weak) components at time $t_{k}$ is $N_{k}(t)\left(W_{k}(t)\right)$. $\beta\left(t_{k}\right) \& \alpha\left(t_{k}\right)$ can be estimated through either a test procedure (see [16] as an example) or a statistical method (see [21] as an example).

In selecting an optimal burn-in time or optimal times from the candidates $\left\{t_{1}, \ldots, t_{n}\right\}$ using ROC analysis, the following four scenarios are considered:

(1) $\frac{C_{\beta}}{C_{\alpha}} \& p$ can be specified,

(2) $\frac{C_{\beta}}{C_{\alpha}} \& p$ cannot be specified,

when the following two cases are taken into consideration,

(1) $N_{k}(t) \& W_{k}(t)$ can be estimated,

(2) $N_{k}(t) \& W_{k}(t)$ cannot be estimated, but $\alpha\left(t_{k}\right) \& \beta\left(t_{k}\right)$ are available.

Obviously, when $N_{k}(t) \& W_{k}(t)$ are available, ROC analysis for continuous models will be used. When only $\alpha\left(t_{k}\right) \& \beta\left(t_{k}\right)$ are available, ROC analysis for discrete models will be used. 


\section{Determining the optimal burn-in time un- der different scenarios}

In general, if the burn-in aims to remove the weak population from the entire, then two objectives are set. The first is to remove all of the weak components through burn-in, as weak components survived from burn-in may incur larger costs during the warranty time. The second is to keep all of the strong components within burn-in, as incorrect burn-in may cause strong components failed within burn-in time, and incur unnecessary costs. In achieving these two goals, one hopes that in Table $2, \beta(t)$ can be minimized, and $1-\alpha(t)$ can be maximized. ROC analysis can help to achieve these goals.

Recall the expected mis-classification cost in (2):

$$
C(t)=C_{\alpha}(1-p) \alpha(t)+C_{\beta} p \beta(t)
$$

Assume $\alpha(t)=N(t) \& \beta(t)=1-W(t)$, where $N(t) \& W(t)$ are the cumulative distribution functions.

Denote $x=\beta(t)=1-W(t)$, and $y=1-\alpha(t)=1-N(t)$. Then

$$
y=1-N\left(W^{-1}(1-x)\right)
$$

We can draw a curve, or an ROC curve on the ROC space, with $\beta(t)$ on the $\mathrm{X}$-axis, and $1-\alpha(t)$ on the $\mathrm{Y}$-axis (where $0 \leq t<\infty$ ).

\subsection{Precise values of $\frac{C_{\beta}}{C_{\alpha}} \& p$ are given}

The luckiest scenario is that precise values of $\frac{C_{\beta}}{C_{\alpha}} \& p$ are specified. However, in order to obtain the optimal burn-in time, one should estimate the life distributions of the strong \& weak populations. 


\subsection{1 $N_{k}(t)$ (or $\left.W_{k}(t)\right)$ are from an identical distribution}

Suppose that $N_{k}(t) \& W_{k}(t)$ are from an identical distributions, say, $N(t) \&$ $W(t)$ ), respectively. This assumption has been made by most authors (see [16, 17] for example). Replacing $\beta(t) \& \alpha(t)$ with $1-N(t) \& W(t)$ in (6), respectively, we obtain

$$
C(t)=C_{\alpha}(1-p) N(t)+C_{\beta} p(1-W(t))
$$

Based on (8), the optimal burn-in time should satisfy the following equation,

$$
\frac{d C(t)}{d t}=C_{\alpha}(1-p) \frac{d N(t)}{d t}-C_{\beta} p \frac{d W(t)}{d t}=0 .
$$

Therefore the optimal burn-in time, $t^{*}$, satisfies

$$
\frac{\frac{d N(t)}{d t}}{\frac{d W(t)}{d t}}=\frac{p C_{\beta}}{(1-p) C_{\alpha}} .
$$

Equation 10 can only be used when $\frac{p C_{\beta}}{(1-p) C_{\alpha}}$ and $N(t)$, and $W(t)$ are available. This approach has been utilized by [16, 20, 19, 21].

One can select the optimal burn-in time, $t_{k}^{*}$, that is close to $t^{*}$.

\subsubsection{Only $\alpha\left(t_{k}\right) \& \beta\left(t_{k}\right)$ are available}

Denote $l=\frac{p C_{\beta}}{(1-p) C_{\alpha}}$. According to $\sqrt{2}$, then one can choose the optimal burn-in time, $t^{*} \in\left\{t_{1}, t_{2}, \ldots, t_{n}\right\}$, that satisfies

$$
\alpha\left(t^{*}\right)+l \beta\left(t^{*}\right)=\min _{1 \leq k \leq n}\left(\alpha\left(t_{k}\right)+l \beta\left(t_{k}\right)\right) .
$$

\subsection{Precise values of $\frac{C_{\beta}}{C_{\alpha}} \& p$ are not given}

The unluckiest scenario is that no information about $\frac{C_{\beta}}{C_{\alpha}} \& p$ is available, which might rarely happen in practice. However, it is useful for practitioners, 
for example, product designers, to obtain more knowledge about the burn-in cost \& burn-in time of their products.

Following (7), at different check points $t_{k}$, the expected cost in value $x\left(=1-W_{k}(t)\right)$ is given by

$$
\begin{aligned}
C_{E}\left(t_{k}\right) & =\int_{0}^{1}\left(C_{\alpha}(1-p)(1-y)+C_{\beta} p x\right) d x \\
& =\frac{1}{2} p C_{\beta}+(1-p) C_{\alpha}-(1-p) C_{\alpha} \int_{0}^{1} y d x,
\end{aligned}
$$

where $y=1-N_{k}\left(W_{k}^{-1}(1-x)\right)$. Therefore, minimizing the quantity $C_{E}\left(t_{k}\right)$ is equivalent to maximizing $\int_{0}^{1} y d x$, which is the AUC (Area Under the ROC Curve) of $y=1-W_{k}\left(N_{k}^{-1}(1-x)\right)$, that is

$$
\operatorname{AUC}\left(t_{k}\right)=\int_{0}^{1} y d x
$$

Hence, 12 can be re-written as

$$
C_{E}\left(t_{k}\right)=\frac{1}{2} p C_{\beta}+(1-p) C_{\alpha}-(1-p) C_{\alpha} \operatorname{AUC}\left(t_{k}\right) .
$$

\subsection{1 $\quad N_{k}(t) \& W_{k}(t)$ are available}

The AUC can be used when one wants to compare the performance of burn-in in different time $t_{k}$.

Plotting $x=1-W_{k}(t)$ on the $\mathrm{X}$-axis, and $y=1-N_{k}(t)$ on the $\mathrm{Y}$-axis, one can draw ROC curves $y=1-N_{k}\left(W_{k}^{-1}(1-x)\right)$. By comparing the AUC values of the ROC curves at times $t_{k}(k=1, \ldots, n)$, one can choose optimal burn-in times with the largest AUC values.

However, the $p$ values in (14) might be different at different time $t_{k}(k=$ $1, \ldots, n)$ (see [21] for example). The following Lemma can be used to compare $C_{E}\left(t_{k}\right)$ values with different $p$ 's. 
Lemma 2. Suppose the probabilities of mis-classifying a strong (weak) component as weak (strong) are $1-y_{i}\left(x_{i}\right)$ at time $t_{i}$, and $1-y_{j}\left(x_{j}\right)$ at time $t_{j}$, respectively. Then

1. $C_{E}\left(t_{i}\right)>C_{E}\left(t_{j}\right)$ if $\hat{p}_{i}>\hat{p}_{j}$, and 1-(1-AUC(t $\left.t_{i}\right) \rho<A U C\left(t_{j}\right)$, and

2. $C_{E}\left(t_{i}\right)<C_{E}\left(t_{j}\right)$ if $\hat{p}_{i}<\hat{p}_{j}$, and 1-(1-AUC(t $\left.t_{i}\right) \rho>A U C\left(t_{j}\right)$,

where $\rho=\frac{\left(1-\hat{p}_{i}\right) \hat{p}_{j}}{\hat{p}_{i}\left(1-\hat{p}_{j}\right)}$.

Proof.

$$
\begin{aligned}
C_{E}\left(t_{i}\right) & =\int_{0}^{1}\left(C_{\alpha}\left(1-\hat{p}_{i}\right)\left(1-y_{i}\right)+C_{\beta} \hat{p}_{i} x\right) d x \\
& =\frac{\hat{p}_{i}}{\hat{p}_{j}} \int_{0}^{1}\left(C_{\alpha}\left(1-\hat{p}_{j}\right)\left(1-\left(1-\rho+\rho y_{i}\right)\right)+C_{\beta} \hat{p}_{j} x\right) d x \\
& =\frac{\hat{p}_{i}}{\hat{p}_{j}}\left\{\frac{1}{2} \hat{p}_{j} C_{\beta}+\left(1-\hat{p}_{j}\right) C_{\alpha}-\left(1-\hat{p}_{j}\right) C_{\alpha} \int_{0}^{1}\left(1-\rho+\rho y_{i}\right) d x\right\} \\
& =\frac{\hat{p}_{i}}{\hat{p}_{j}}\left\{\frac{1}{2} \hat{p}_{j} C_{\beta}+\left(1-\hat{p}_{j}\right) C_{\alpha}-\left(1-\hat{p}_{j}\right) C_{\alpha}\left(1-\left(1-A U C\left(t_{i}\right)\right) \rho\right)(\neq 5)\right.
\end{aligned}
$$

Hence,

$$
\frac{C_{E}\left(t_{i}\right)}{C_{E}\left(t_{j}\right)}=\frac{p_{i}}{\hat{p}_{j}} \frac{\frac{1}{2} \hat{p}_{j} C_{\beta}+\left(1-\hat{p}_{j}\right) C_{\alpha}-\left(1-\hat{p}_{j}\right) C_{\alpha}\left(1-\left(1-A U C\left(t_{i}\right)\right) \rho\right)}{\frac{1}{2} \hat{p}_{j} C_{\beta}+\left(1-\hat{p}_{j}\right) C_{\alpha}-\left(1-\hat{p}_{j}\right) C_{\alpha} A U C\left(t_{j}\right)} .
$$

If $\hat{p}_{i}>\hat{p}_{j}$, and 1- $\rho\left(1-\operatorname{AUC}\left(t_{i}\right)\right)<A U C\left(t_{j}\right)$, we have $\frac{C_{E}\left(t_{i}\right)}{C_{E}\left(t_{j}\right)}>1$, or $C_{E}\left(t_{i}\right)>$ $C_{E}\left(t_{j}\right)$. If $\hat{p}_{i}<\hat{p}_{j}$, and $1-\rho\left(1-\operatorname{AUC}\left(t_{i}\right)\right)>A U C\left(t_{j}\right)$, we have $\frac{C_{E}\left(t_{i}\right)}{\left.C_{E}\left(t_{j}\right)\right]}<1$, or $C_{E}\left(t_{i}\right)<C_{E}\left(t_{j}\right)$.

\subsubsection{Only $\alpha\left(t_{k}\right) \& \beta\left(t_{k}\right)$ are available}

When the $p$ values in (6) are equivalent at different time $t_{k}(k=1, \ldots, n)$, the following approach can be used. 
Plot $n$ points $\left(\beta\left(t_{k}\right), 1-\alpha\left(t_{k}\right)\right)(k=1, \ldots, n)$ on the ROC space, then construct line $L_{k}$ by linking points $(0,0),\left(\beta\left(t_{k}\right), 1-\alpha\left(t_{k}\right)\right)$, and line $L_{k}^{\prime}$ by linking points $\left(\beta\left(t_{k}\right), 1-\alpha\left(t_{k}\right)\right)$, and $(1,1) . L_{k}, L_{k}^{\prime}$, a line linked from $(0,0)$ to $(1,0))$, and a line linked from $(1,0)$ to $(1,1)$, construct a rectangle. The area of this rectangle represents the performance of burn-in at time $t_{k}$. The time point with the largest area among the burn-in times $\left(t_{1}, \ldots, t_{n}\right)$ is the optimal time burn-in time. Table 5 presents the algorithm of searching the optimal burn-in time $t^{*}$.

When the $p$ values in (6) are different with time $t_{k}(k=1, \ldots, n)$, the following approach can be used. Selecting the largest value, $p^{*}$, from $p_{k}$ $(k=1,2, \ldots, n)$, for a time point $t_{k}$,

$$
\begin{aligned}
C\left(t_{k}\right) & =C_{\alpha}\left(1-p_{k}\right) \alpha\left(t_{k}\right)+C_{\beta} p_{k} \beta\left(t_{k}\right) \\
& =C_{\alpha}\left(1-p^{*}\right)\left(\frac{\left(1-p_{k}\right)}{1-p^{*}} \alpha\left(t_{k}\right)\right)+C_{\beta} p^{*}\left(\frac{p_{k}}{p^{*}} \beta\left(t_{k}\right)\right) .
\end{aligned}
$$

Hence the algorithm from Table 5 can be used to search the optimal points

among points $\left(\frac{p_{k}}{p^{*}} \beta\left(t_{k}\right), 1-\frac{1-p_{k}}{1-p^{*}} \alpha\left(t_{k}\right)\right)$. This approach subjects to $\frac{\left(1-p_{k}\right)}{1-p^{*}} \alpha\left(t_{k}\right)<$ 1.

\subsection{ROC analysis to models developed by Tseng et al. $[20,21]$}

Tseng et al. [20, 21] obtain (10) by minimizing (2). Then they determine the optimal burn-in time by choosing a time point from $t_{k}$ (for example, $t_{k}=2,4,8,16,32,64,128$ in Tseng et al. [21]) that minimizes the following equation.

$$
C_{1}(t)=C_{\alpha}(1-p) \alpha(t)+C_{\beta} p \beta(t)+C_{\text {ope }} t+C_{\text {mea }}\left(\log _{2} t+2\right),
$$


where $C_{\text {ope }} \& C_{\text {mea }}$ denote unit cost of operating the degradation test and the cost of measuring data on a unit, respectively.

Tseng \& Peng [21] give the mean and variance of $g_{i}(t)+\sigma B(\tau(t))(i=1,2)$ are $g_{i}(t) \& \sigma^{2} \tau(t)$, respectively. The mean \& variance of $\int_{0}^{t} g_{i}(s)+\sigma B(\tau(s)) d s$ $(i=1,2)$ are $\int_{0}^{t} g_{i}(s) d s \& 2 \sigma^{2} \int_{0}^{t}(t-s) \tau(s) d s$, respectively.

Denote

$$
\Delta_{0}(t)=\frac{g_{2}(t)-g_{1}(t)}{\sqrt{\sigma^{2} \tau(t)}},
$$

and

$$
\Delta_{1}(t)=\frac{\int_{0}^{t}\left(g_{2}(s)-g_{1}(s)\right) d s}{\sqrt{2 \sigma^{2} \int_{0}^{t}(t-s) \tau(s) d s}} .
$$

According to Hanley \& McNeil [25], the values of the AUC for the NCD \& CD method are given by

$$
\mathrm{AUC}_{0}=\Phi\left(\frac{\Delta_{0}}{\sqrt{2}}\right)
$$

and

$$
\mathrm{AUC}_{1}=\Phi\left(\frac{\Delta_{1}}{\sqrt{2}}\right)
$$

respectively. Here $\Phi$ denotes the cumulative distribution function of a standard normal distribution.

Based these two AUC values, we have

Lemma 3. If $\Delta_{0}(t)<\Delta_{1}(t)$ for all $t$, then the $C D$ method is preferable to the NCD method, and vice versa.

Proof. If $\Delta_{0}(t)<\Delta_{1}(t)$ for all $t$, then from (19) \& (20), $\mathrm{AUC}_{0}>\mathrm{AUC}_{1}$. According to (14), the larger value of the AUC is, the smaller the value of the expected mis-classification cost. 
The results from Lemma 2 is the same as the results of Theorem 3 in the paper [21] which, however, takes a long proof.

For a fixed $t$, the probabilities of mis-classifying are

$$
\alpha_{0}=\Phi\left(\frac{\xi_{0}^{*}(t)-g_{2}(t)}{\sqrt{\sigma^{2} \tau(t)}}\right), \beta_{0}=1-\Phi\left(\frac{\xi_{0}^{*}(t)-g_{1}(t)}{\sqrt{\sigma^{2} \tau(t)}}\right),
$$

for the NCD method and

$$
\alpha_{1}=\Phi\left(\frac{\xi_{1}^{*}(t)-\int_{0}^{t} g_{2}(s) d s}{\sqrt{2 \sigma^{2} \int_{0}^{t}(t-s) \tau(s) d s}}\right), \beta_{1}=1-\Phi\left(\frac{\xi_{1}^{*}(t)-\int_{0}^{t} g_{1}(s) d s}{\sqrt{2 \sigma^{2} \int_{0}^{t}(t-s) \tau(s) d s}}\right)
$$

for the CD method, respectively.

For time point $t_{k}$, one can draw an ROC curve that represents the change of the values $1-\alpha\left(t_{k}\right)$ over $\beta\left(t_{k}\right)$. Given $n$ time points, $n$ curves can be drawn, and $n$ values of AUC of the corresponding curves can be calculated.

If $p, \frac{C_{o p e}}{C_{\alpha}} \frac{C_{m e a}}{C_{\alpha}}$ can be estimated, and

$$
\alpha^{\prime}(t)=\alpha(t)+\frac{C_{\text {ope }} t+C_{\text {mea }}\left(\log _{2} t+2\right)}{C_{\alpha}(1-p)}<1,
$$

then $C_{1}(t)$ in 18$)$ can be re-written as

$$
C_{1}(t)=C_{\alpha}(1-p) \alpha^{\prime}(t)+C_{\beta} p \beta(t)
$$

One can draw an ROC curve by plotting $\beta(t)$ on the $\mathrm{X}$-axis, and $1-\alpha^{\prime}(t)$ on the Y-axis, and selects possible optimal burn-in times on the ROC convex hull. (24) hold only if (23) is held. Examples from [19, 21] satisfies the condition 23 .

As the values of $\alpha(t)$ might be so small that all of points $(\beta(t), 1-\alpha(t))$ are close to the line $y=1$, which makes it hard to compare the points and 
draw an ROC convex hull. However, if one constitutes a new ROC space properly, it is possible to magnify the space, which is the part of our interest. The following Lemma proves that such procedure can not change the relative positions among the points in the two ROC spaces.

Lemma 4. If we define a new $R O C$ space as $\left(X_{n}, Y_{n}\right)$ based on the original ROC space $\left(X_{o}, Y_{o}\right)$, set $X_{n}=\lambda X_{o} \& Y_{n}=Y_{o}-1+\lambda$ with $0<\lambda \leq 1$, $\left(\beta\left(t_{k}\right), 1-\alpha\left(t_{k}\right)\right)$ on the ROC convex hull in the original $R O C$ space $\left(X_{o}, Y_{o}\right)$ will be still on the ROC convex hull in the new $R O C$ space $\left(X_{n}, Y_{n}\right)$.

Proof. If the angle between the $\mathrm{X}$-axis and the line constituted by linking points $\left(\beta\left(t_{i}\right), 1-\alpha\left(t_{i}\right)\right) \&\left(\beta\left(t_{j}\right), 1-\alpha\left(t_{j}\right)\right)$ does not change, or changes with an identical proportion from the original ROC space $\left(X_{o}, Y_{o}\right)$ to the new ROC space $\left(X_{n}, Y_{n}\right)$, then points on an ROC convex hull in the original ROC space will still on an ROC convex hull in the new ROC space. The angle made in the orignal ROC space can be expressed as $\frac{\alpha\left(t_{i}\right)-\alpha\left(t_{j}\right)}{\beta\left(t_{j}\right)-\beta\left(t_{i}\right)}$. Hence, we have

$$
\begin{aligned}
\frac{\alpha\left(t_{o i}\right)-\alpha\left(t_{o j}\right)}{\beta\left(t_{o j}\right)-\beta\left(t_{o i}\right)} & =\frac{\alpha\left(t_{n i}\right)-1+\lambda-\alpha\left(t_{n j}\right)+1-\lambda}{\lambda \beta\left(t_{n j}\right)-\lambda \beta\left(t_{n i}\right)} \\
& =\frac{1}{\lambda} \frac{\alpha\left(t_{n i}\right)-\alpha\left(t_{n j}\right)}{\beta\left(t_{n j}\right)-\beta\left(t_{n i}\right)}
\end{aligned}
$$

where $\alpha\left(t_{o i}\right), \alpha\left(t_{o j}\right), \beta\left(t_{o i}\right)$, and $\beta\left(t_{o j}\right)$ are for the original ROC space, and $\alpha\left(t_{n i}\right), \alpha\left(t_{n j}\right), \beta\left(t_{n i}\right)$, and $\beta\left(t_{n j}\right)$ are for the new ROC space.

This proves that the procedure does not change the relative positions of the points in the ROC spaces. 


\section{$5 \quad$ Illustrative examples}

This section gives one numerical example to illustrate the validity of the approaches introduced in the paper. We first determine the optimal burn-in times for the cases when $W(t) \& N(t)$ are available, but $C_{\alpha}, C_{\beta} \& p$ are not available. It uses ROC analysis to compare the expected mis-classification cost in (14) when the distributions $W(t) \& N(t)$ are available. Lemma 1 re-exams the illustrative examples from [21] are also used in this example. Then we assume that further information including $p, C_{\beta} \& C_{\alpha}$ are available, and select optimal burn-in times on the ROC convex hull for the example given by [21].

Tseng \& Peng [21] give an illustrative example on obtaining the optimal burn-in time. It optimizes the burn-in time for a kind of new LED lamps which are developed for copy/fax machines. The cost criterion is set as in (18). They investigate two cases: $\gamma=0.6 \& \gamma=0.1$. For simplicity, here we only study the case with $\gamma=0.6$. Relevant parameters shown in Table 6 are given in [21].

Using Lemma 2 , we set $i=1,2,3,4,5,6,7, \hat{p}_{j}=p_{6}=0.0569$ for the NCD method. $p_{6}$ is the smallest one, i.e., $\hat{p}_{j}<\hat{p}_{i}$, whereas the AUC value at $t_{6}(0.8467)$ is larger than that at $t_{7}(0.8439)$, therefore, $C_{E}\left(t_{6}\right)>C_{E}\left(t_{7}\right)$ (see Table 3). This suggests $t=128$ should not be considered as an optimal burn-in time with any costs of $C_{\alpha}, C_{\beta}, C_{\text {mea }}$, and $C_{\text {opt }}$.

Similarly, for the CD method, we have $p_{6}<p_{7} \& C_{E}\left(t_{6}\right)>C_{E}\left(t_{7}\right)$ (see Table 3). According to Lemma 1, we suggest $t=128$ should not be considered as an optimal burn-in time with any costs of $C_{\alpha}, C_{\beta}, C_{m e a}$, and $C_{\text {opt }}$. 
Table 3 also tells us that the CD method is superior to the NCD method because the AUC value at each time point of the CD method is larger than that of the NCD method.

All of the above results in this Example are reached when no cost information is used. This can be verified as follows, when cost information about $C_{\alpha}, C_{\beta}, C_{\text {opt }}$, and $C_{\text {mea }}$ are available.

The cost of $C_{\text {opt }} t+C_{\text {mea }}\left(\log _{2} t+2\right)$ at time $t_{i}(i=1, \ldots, 7)$ are 0.34, 0.48, $0.66,0.92,1.34,2.08$, and 3.46 , respectively. Comparing these cost with cost in Table 6 of the CD \& NCD methods, we can draw two curves of misclassification cost versus time $t_{i}$, as shown in Figure 6, which is obtained based on removing the operating \& measuring cost from the total burn-in cost. Although the mis-classification cost at time $t_{i}(i=1, \ldots, 5)$ is larger than at time $t_{6}$ (see Figure 6), we cannot conclude that burn-in cost at time $t_{i}(i=1, \ldots, 5)$ is less than time $t_{6}$. This is because the operating \& measuring cost is cheaper at time $t_{i}(i=1, \ldots, 5)$. At time $t_{7}$, however, both the misclassification cost, and operating \& measuring cost are larger than time $t_{6}$, it is concluded that the burn-in cost at $t_{6}=64$ is less than that at time $t_{7}=128$. This verifies that our results are correct.

Table 3: AUC values for $\gamma=0.6$.

\begin{tabular}{l|ccccccc}
\hline & $t=2^{1}$ & $t=2^{2}$ & $t=2^{3}$ & $t=2^{4}$ & $t=2^{5}$ & $t=2^{6}$ & $t=2^{7}$ \\
\hline AUC (NCD method) & 0.9254 & 0.8963 & 0.8761 & 0.8599 & 0.8554 & 0.8467 & 0.8439 \\
\hline AUC (CD method) & 0.9443 & 0.9108 & 0.8900 & 0.8698 & 0.8652 & 0.8581 & 0.8521 \\
\hline
\end{tabular}

In the following, we have used cost information including $C_{\alpha} \& C_{\beta}$.

We use the above example to draw a ROC convex hull (see Figure 7) for searching optimal burn-in times for the case $\gamma=0.6$. Setting $p^{*}=0.0646$, 


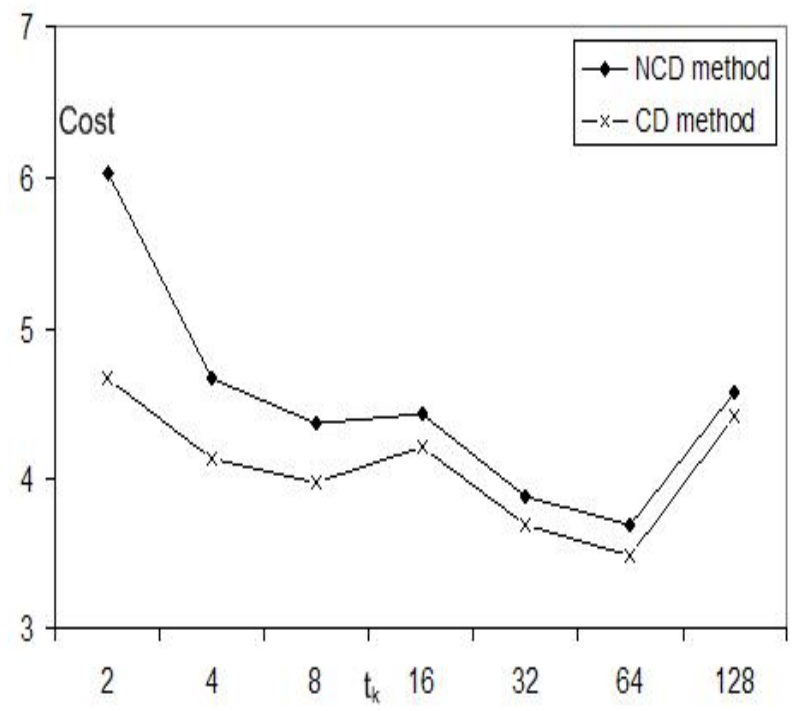

Figure 6: Misclassification cost at different times.

and using (17) based on quantities of $\alpha_{i} \& \beta_{i}(i=0,1)$ from Table 6, one can obtain new probabilities of mis-classifying as shown in Table 4.

From Figure 7, only time point $t_{6}$ for the $\mathrm{CD}$ method is on the ROC convex hull, which matches the above analytical result, that is, $t_{6}$ is the optimal burn-in time for the method CD if the operating \& measuring cost is not considered.

Table 4: $1-\alpha^{\prime} \& \beta^{\prime}(\gamma=0.6$ with NCD \& CD methods).

\begin{tabular}{l|l|lllllll}
\hline & & $t=2^{1}$ & $t=2^{2}$ & $t=2^{3}$ & $t=2^{4}$ & $t=2^{5}$ & $t=2^{6}$ & $t=2^{7}$ \\
\hline \multirow{3}{*}{$\mathrm{NCD}$} & $\beta^{\prime}$ & 0.7414 & 0.5995 & 0.5770 & 0.6036 & 0.5269 & 0.5016 & 0.5390 \\
& $1-\alpha^{\prime}$ & 0.9717 & 0.9806 & 0.9834 & 0.9850 & 0.9864 & 0.9872 & 0.9860 \\
\hline \multirow{3}{*}{$\mathrm{CD}$} & $\beta^{\prime}$ & 0.5411 & 0.5120 & 0.5110 & 0.5622 & 0.4927 & 0.4646 & 0.6069 \\
& $1-\alpha^{\prime}$ & 0.9749 & 0.9811 & 0.9834 & 0.9846 & 0.9862 & 0.9870 & 0.9855 \\
\hline
\end{tabular}




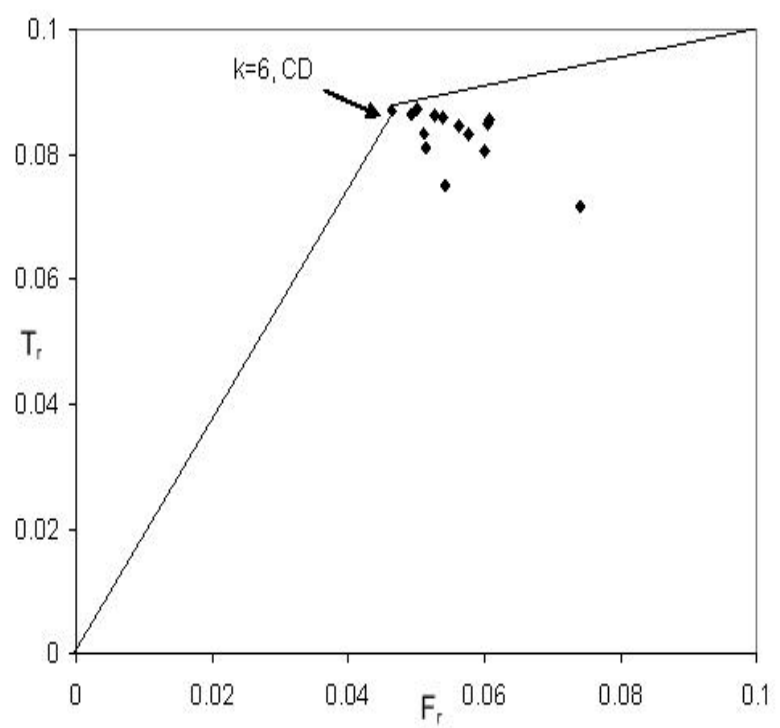

Figure 7: ROC space with $\gamma=0.6$.

\section{Conclusions}

When a population of components consists of weak \& strong subpopulations, to remove the weak subpopulation can improve the reliability of the population. This goal can be achieved by many approaches, from which optimal approaches can be selected by minimizing the expected mis-classification cost. When little information about mis-classification cost \& the proportion of weak \& strong components are available, ROC (receiver operating characteristic) analysis is an ideal tool for selecting possible optimal models \& discard suboptimal approaches. This approach is introduced in this paper.

Burn-in is a widely used procedure to remove weak components for a mixture of strong \& weak populations. However, performing burn-in for such a population cannot ensure that all of weak components would be removed (or all of strong components would be remained), which will incur losses. In 
order to minimize the losses, one can optimize the burn-in time. Determining optimal burn-in times might have several steps such as estimating life distributions of the components, and various costs incurred. The proportion of the weak (or strong ) population, and associated costs, should be given in order to estimate the expected burn-in cost for the entire population correctly. However, in reality, it can be to hard to obtain the life distributions of the weak (or strong ) population, and costs incurred when a strong (or weak) component fails through (or survives) burn-in, to optimize burn-in time in such scenarios is useful and helpful for practical use. However, little research can be found in the reliability literature to tackle such problems, which was discussed in this paper.

The main contributions of the paper are

- weak \& strong sub-populations are classified using ROC analysis;

- an optimization criterion for minimizing the burn-in error is introduced; and

- an algorithm is presented to find the optimal burn-in time.

\section{Acknowledgement}

We are grateful to the reviewers, and the Associate Editor for their helpful comments \& suggestions, which have resulted in a number of improvements in the paper. 


\section{References}

[1] J. P. Egan, Signal detection theory and ROC analysis. Academic Press, 1975 .

[2] D. M. Green and J. A. Swets, Signal detection theory and psychophsics. New York: Wiley, 1966.

[3] C. E. Metz, "Some practical issues of experimental design and data analysis in radiological ROC studies," Investigation Radiology, vol. 24, pp. 234-245, 1989.

[4] J. K. Hsiao, J. J. Bartko, and W. Z. Potter, "Diagonising diagnoses receiver operating characteristic methods and psychiatry," Archives of General Psychiatry, vol. 46, pp. 664-667, 1989.

[5] C. Nockmann, H. Heidt, and N. Thomsen, "Reliability in NDT: ROC study of radiographic weld inspections," Nondestructive Testing and Evaluation International, vol. 24, pp. 235-245, 1991.

[6] F. Provost and T. Fawcett, "Robust classification for imprecise environments," Machine Learning, vol. 42, pp. 203-231, 2001.

[7] J. Mi, "Bathtub failure rate and upside-down bathtub mean residual life," IEEE Transaction on Reliability, vol. 44, no. 3, pp. 388-391, 1995.

[8] C. E. Ebeling, An introduction to reliability and maintainability engineering. McGraw-Hill Companies, 1997.

[9] J. H. Cha, "On optimal burn-in procedures - a generalized model," IEEE Transactions on Reliability, vol. 54, pp. 198-205, 2005. 
[10] S. H. Sheu and Y. H. Chien, "Minimizing cost-functions related to both burn-in and field-operation under a generalized model," IEEE Transactions on Reliability, vol. 53, pp. 435-439, 2004.

[11] S. J. Bae and P. H. Kvam, "A change-point analysis for modeling incomplete burn-in for light displays," IIE Transactions, vol. 38, pp. 489-498, 2006 .

[12] J. H. Cha, "An extended model for optimal burn-in procedures," IEEE TRANSACTIONS ON RELIABILITY, vol. 55, no. 2, pp. 189-198, 2006.

[13] K. O. Kim and W. Kuo, "Some consideration on system burn-in," IEEE Transactions on Reliability, vol. 54, no. 2, pp. 207-214, 2005.

[14] J. H. K. Kao, "A graphical estimation of mixed weibull parameters in life testing of electron tubes," Technometrics, vol. 1, pp. 389-407, 1959.

[15] T. J. Boardman and R. E. Colvert, "Estimation in the piece-wise constant harzard rate model when the data are grouped," Communications in Statistics, vol. 8, no. 5, pp. 417-432, 1978.

[16] K. N. Kim, "Optimal burn-in for minimizing cost and multi-objectives," Microelectronics and Reliability, vol. 38, no. 10, pp. 1577-1583, 1998.

[17] D. Perlstein, W. H. Jarvis, and T. A. Mazzuchi, "Bayesian calculation of cost optimal burn-in test durations for mixed exponential populations," Reliability Engineering and System Safety, vol. 72, no. 3, pp. 265-273, 2001. 
[18] N. Singh and S. Bhattacharya, "Bayesian analysis of the mixture of exponential failure distributions," Microelectronics and Reliability, vol. 33, no. 8, pp. 1113-1117, 1993.

[19] S. T. Tseng, J. Tang, and I. H. Ku, "Determination of burn-in parameters and residual life for highly reliable products," Naval Research Logistics, vol. 50, pp. 1-14, 2002.

[20] S. T. Tseng and J. Tang, "Optimal burn-in time for highly reliable products," International Journal of Industrial Engineering, vol. 8, pp. 329-338, 2001.

[21] S. T. Tseng and C. Y. Peng, "Optimal burn-in policy by using an integrated wiener process," IIE Transaction, vol. 36, pp. 1161-1170, 2004.

[22] P. Flach and S. Wu, "Repairing concavities in ROC curves," in Proceeding of 19th International Conference of Joint Conference of Artificial Intelligence, Edinburgh, 2005.

[23] S. Wu and P. Flach, "A scored metric for classifier evaluation and selection," in $R O C M L, 2005$.

[24] M. H. Zweig and G. Campbell, "Receiver-operating characteristic (ROC) plots: a fundamental evaluation tool in clinical medicine," Clinical Chemistry, vol. 39, pp. 561-577, 1993.

[25] J. A. Hanley and B. J. McNeil, "A method of comparing the areas under receiver operating characteristic curves derived from the same cases," Radiology, vol. 148, pp. 839-843, 1983. 
Table 5: Searching the optimal burn-in time $t^{*}$.

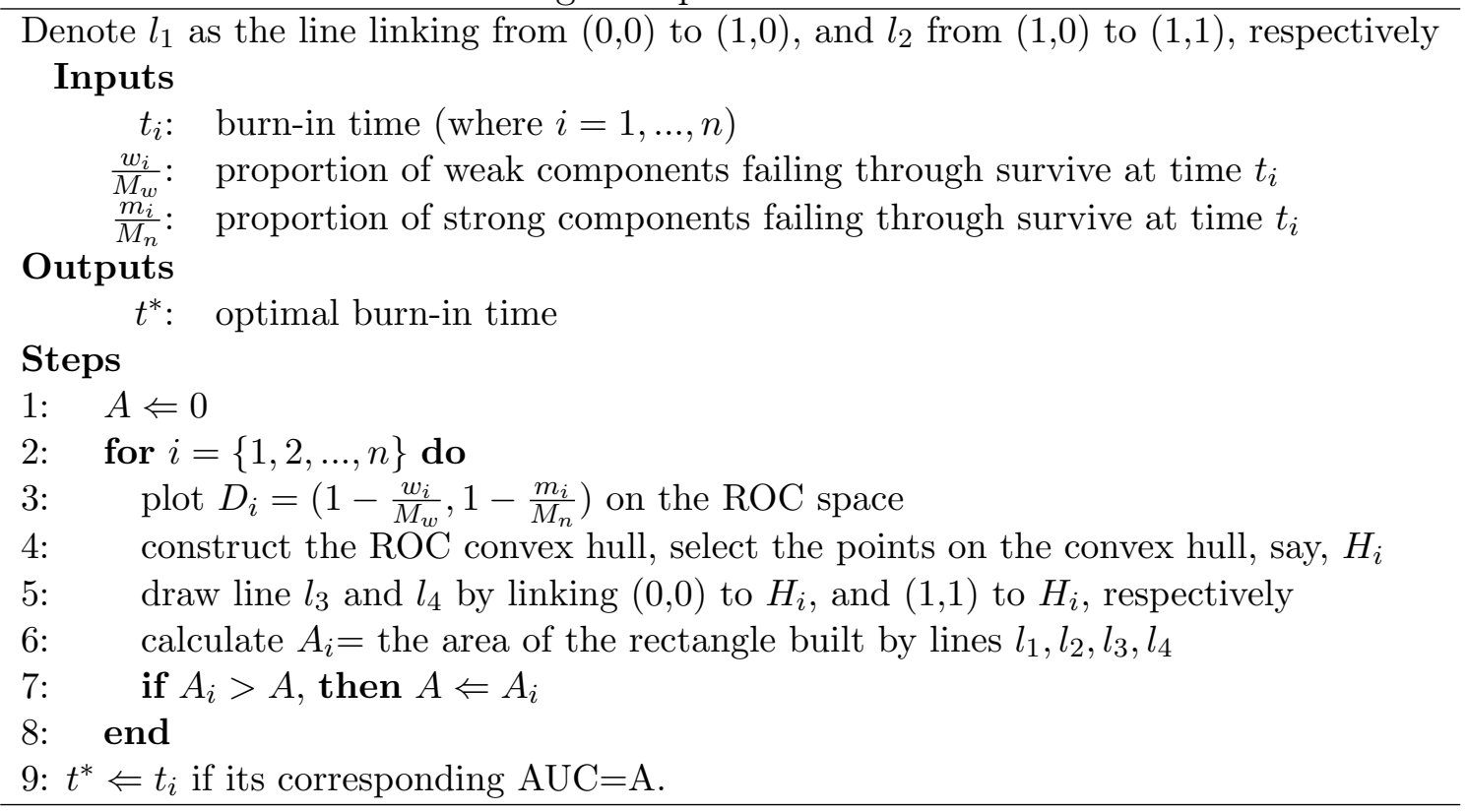


Table 6: Parameters estimated when $\gamma=0.6$ (see [21]).

\begin{tabular}{llllllll}
\hline & $t=2^{1}$ & $t=2^{2}$ & $t=2^{3}$ & $t=2^{4}$ & $t=2^{5}$ & $t=2^{6}$ & $t=2^{7}$ \\
\hline$\hat{\delta}$ & 0.3628 & 0.3788 & 0.3758 & 0.3740 & 0.3781 & 0.3782 & 0.3779 \\
$\hat{\gamma}$ & 0.8231 & 0.6846 & 0.6496 & 0.5988 & 0.5939 & 0.6042 & 0.5862 \\
$\hat{\eta}_{1}$ & 0.0255 & 0.0258 & 0.0254 & 0.0247 & 0.0245 & 0.0243 & 0.0231 \\
$\hat{\eta}_{2}$ & 0.0164 & 0.0167 & 0.0168 & 0.0169 & 0.0169 & 0.0170 & 0.0170 \\
$\hat{\sigma}$ & 0.0043 & 0.0046 & 0.0047 & 0.0049 & 0.0049 & 0.0049 & 0.0050 \\
$\hat{p}$ & 0.1102 & 0.0798 & 0.0700 & 0.0659 & 0.0597 & 0.0569 & 0.0646 \\
NCD method & & & & & & & \\
\hline$\alpha_{0}$ & 0.0297 & 0.0197 & 0.0167 & 0.0150 & 0.0135 & 0.0127 & 0.0140 \\
$\beta_{0}$ & 0.4346 & 0.4853 & 0.5325 & 0.5917 & 0.5701 & 0.5695 & 0.6390 \\
Cost & 6.368 & 5.146 & 5.023 & 5.343 & 5.224 & 5.771 & 8.028 \\
CD method & & & & & & & \\
\hline$\alpha_{1}$ & 0.0264 & 0.0192 & 0.0167 & 0.0154 & 0.0137 & 0.0129 & 0.0143 \\
$\beta_{1}$ & 0.3172 & 0.4145 & 0.4716 & 0.5511 & 0.5331 & 0.5275 & 0.6069 \\
Cost & 5.011 & 4.607 & 4.640 & 5.122 & 5.037 & 5.568 & 7.868 \\
\hline
\end{tabular}

Authors:

Shaomin Wu received his M.Sc. \& Ph.D. in applied mathematics from Southeast University in P.R. China. His research interests include system reliability analysis, statistical process control, data mining, and machine learning. He has published over 40 papers in conferences \& journals such as "IEEE Transactions on Reliability", "Microelectronics \& Reliability", "Reliability Engineering \& System Safety" and "Computers \& Industrial Engineering". He is on the editorial board of journal Asian Journal of Information Management.

Min Xie is a professor at Department of Industrial \& Systems Engineering, National University of Singapore. He received his PhD in Quality Technology from Linkoping University in 1987. He has been active in research in the area of reliability, quality, and engineering statistics. He is on the editorial board of several international journals. Prof Xie is an author or co-author 
of numerous papers and six books including Computing Systems Reliability published by Kluwer, and Weibull Models published by Wiley. He is an Elected Fellow of IEEE. 
of numerous papers and six books including Computing Systems Reliability published by Kluwer, and Weibull Models published by Wiley. He is an Elected Fellow of IEEE. 